\title{
Development of Secondary Electron Time Detector for Ion Beams
}

\author{
S. Suzuki*, A. Ozawa, T. Moriguchi, Y. Ichikawa, M. Amano, D. Kamioka, Y. Tajiri, \\ K. Hiraishi, T. Matsumoto \\ Institute of Physics, University of Tsukuba, Ibaraki 305-8571, Japan \\ E-mail: ssuzukietac.tsukuba.ac.jp
}

D. Nagae, Y. Abe, S. Naimi

RIKEN, Nishina Center, Wako, Saitama 351-0198, Japan

\author{
T. Yamaguchi, S. Omika, Z. Ge, N. Tadano, K. Wakayama
}

Department of Physics, Saitama University, Saitama 338-8570, Japan

\section{A. Kitagawa, S. Sato}

National Institute of Radiological Sciences, Chiba 263-8555, Japan

\begin{abstract}
A time detector is under development for the mass measurement with the Rare-RI (radioactive isotope) Ring at the RI beam factory (RIBF) in RIKEN. To perform successful mass measurement, a time detector requires a small energy loss of the ion beams, a good time resolution, and a sufficient detection efficiency. To satisfy these requirements, our time detector utilizes isochronous transport of the secondary electrons emitted from a thin foil. The isochronous transport of the electrons from the foil to the micro-channel plate (MCP) detector is provided by the crossed electromagnetic field. To improve the detection efficiency, the transport time of the electrons is reduced by a factor of two using a strong magnetic field. An experiment to test the performance of the time detector was carried out with heavy ion beams. The time resolution was $\sigma<100 \mathrm{ps}$ with a maximum detection efficiency of $99 \%$ for heavy ion beams. The details of the detector are presented in this contribution.
\end{abstract}

The 26th International Nuclear Physics Conference

11-16 September, 2016

Adelaide, Australia

*Speaker. 


\section{Introduction}

The Isochronous Mass Spectrometry (IMS) with the storage ring has been performed with the ESR in GSI and the CSRe in IMP [1-5]. The combination of a fragment separator and a storage ring enables to measure the masses of short-lived nuclei. In Japan, development of the Rare-RI Ring is in progress at the RIBF in RIKEN. The priority of the Rare-RI Ring is to measure masses of exotic nuclei relevant for the r-process nucleosynthesis using the IMS technique. The mass-tocharge ratio of an exotic nucleus is determined by the its circulation time in the Rare-RI Ring and its velocity [6]. The total circulation time in the Rare-RI Ring is about $1 \mathrm{~ms}$, which is determined by measuring the Time-Of-Flight (TOF) from the injection into the ring to the extraction. The ion velocity is determined by the TOF measured using the transport line before the ring. The time detector which serves both as stop-time detection for the velocity measurement and as start-time detection for the circulation time, is needed for the mass measurement with the Rare-RI Ring. For this purpose, we have developed the detector described in this contribution.

The time detector has to satisfy some requirements for a successful mass measurement with the Rare-RI Ring. The requirements for the time detector are; (i) to achieve a velocity resolution of $10^{-4}$ that leads to the aimed mass precision of $10^{-6}$ [6], the time resolution $\sigma$ should be less than 100 ps since the TOF for transport to the Rare-RI Ring is about $1 \mu \mathrm{s}$; (ii) detection efficiency for the heavy ion should be $100 \%$ for the efficient measurement since the production rate of the exotic rare RI is very low; (iii) the detector should be as thin as possible to minimize the change of particle's velocity in the detector. This change should be less than $10^{-4}$ due to requirement (i).

\section{Detector design}

To satisfy the above-mentioned requirements, the present time detector utilizes the isochronous transport of secondary electrons generated from a thin foil. When an ion passes through the foil, secondary electrons are emitted from both surfaces of the foil as shown in Fig.W. With the crossed electromagnetic field which provides the isochronous transport, the secondary electrons are guided to two MCP detectors placed in forward and backward direction of the ion beam [7].

This technique has been also used in ESR and CSRe to measure turn-by-turn TOF of stored particle in IMS [8-12]. We have also developed the time detector for the Rare-RI Ring [13]. It is reported that the detection efficiency for ion beams of these time detectors is about 70\% [11], with a time resolution of $\sigma \sim 30 \mathrm{ps}$ [12]. The time resolution of the previous detector satisfies the requirement for the mass measurement with the Rare-RI Ring. However, a higher detection efficiency is desired.

The magnetic field in the previous detectors is about 80 Gauss $[10,11]$. As for the present detector, the magnetic field is increased by approximately a factor of two in order to improve the detection efficiency, which strongly depends on the number of detected electrons. In the vertical direction in which the magnetic field is generated (Fig.W), the Lorentz force does not affect the electrons. Hence, the motion of electrons is dispersive in the vertical direction. If the transport time from the foil to the MCP detector is long, some electrons are not detected since they deviate from the effective area of the MCP detector. To solve this problem, the transport time which is roughly given by $\frac{2 \pi m_{e}}{e B}$, should be shorten by increasing the magnetic field. Consequently, the 
magnetic field of the present detector is increased to detect almost electrons by rapid collection of the secondary electrons emitted from the foil. Compared to the previous detector, the transport time from the foil to the MCP detector is about half.

The schematic view of the present detector is shown in Fig.W. In principle, the center-to-center distance from the foil to the MCP detector $d$ is determined by the electric filed $E$ and the magnetic field $B$ as [7]

$$
d=\frac{2 \pi m_{e} E}{e B^{2}}
$$

where $m_{e}$ is mass of an electron and $e$ is elementary charge. To transport all electrons created at any position of the foil to the MCP detector, the center-to-center distance between the foil and the MCP detector should be equal or larger than the foil size. The foil size should be determined by considering the beam size. The present foil size is less than $\phi 25 \mathrm{~mm}$. The center-to-center distances between the foil and each MCP detector are $70 \mathrm{~mm}$ in the present detector.

The various combinations of the magnetic field and the electric field are possible by fixing of the distance from the foil to the MCP detector. In the present detector design, the magnetic field which is generated by a combination of neodymium magnets arrays and iron yokes, is fixed to 170 Gauss, and is about twice as high as that of the former detector to improve the detection efficiency. The electric field should be increased along with an increase of the magnetic field, so as to satisfy to Eq. D. It has been pointed out that the time resolution of the time detector is inversely proportional to the electric field in Ref. [7]. Therefore, the increase of the electric field is important to obtain good time resolution. The electric field in the present detector is provided by the difference of electric potential between the parallel electrodes with gaps of $26 \mathrm{~mm}$, and is variable up to $700 \mathrm{~V} / \mathrm{mm}$. The optimum electric field is determined by measuring the electric field dependence of the detection efficiency for ion beams.

For the foil to generate secondary electrons, carbon of $50 \mu \mathrm{g} / \mathrm{cm}^{2}$ and gold coated $\left(168 \mu \mathrm{g} / \mathrm{cm}^{2}\right)$ per side on $2 \mu \mathrm{m}$ Mylar were employed in the performance tests with $\mathrm{Kr}$ ion beams. The effective sizes of the carbon and the gold coated Mylar are $\phi 15 \mathrm{~mm}$ and $\phi 25 \mathrm{~mm}$, respectively. According to Ref. [14], the secondary electrons yield per impinging ion, emitted from a carbon foil with $\mathrm{Kr}$ ion of $200 \mathrm{MeV} /$ nucleons, is estimated to be 40 . For the gold case, the estimated electrons yield per impinging ion is 180 [15]. Therefore, the detection efficiency for the gold coated Mylar is expected to be higher than that of carbon foil. This detector also has two other foils of $2 \mu \mathrm{m}$ aluminized Mylar at the outer electrodes for the beam entrance and the beam exit. These foils are employed to produce the homogeneous electric field.

Two MCP detectors are used to detect the electrons transported from both foil surfaces. The MCP detectors are made by Hamamatsu Photonics [16]. In this prototype detector, the MCP detectors whose effective area is $\phi 14.5 \mathrm{~mm}$ (model number F4655-10) are used.

To verify whether the magnetic field in the present detector is enough to improve the detection efficiency, electron-tracking simulations were performed using the calculation code. The electron trajectories were obtained by numerical integration using the 4th order Runge-Kutta method. The initial kinetic energy distribution of the electrons emitted from the thin carbon foil [17], and uniform isotropic emission angles of the electrons from the foil were assumed in this simulations. 
A total of 2000 events were simulated using the uniform electromagnetic field. Figure $\square$ shows simulated electron positions at the plane which is in contact with the surface of the MCP detector. The ratios of detected electrons to the emitted from the foil center, using 80 Gauss and 170 Gauss magnetic field strength, were $88 \%$ and $99 \%$, respectively. Nearly all of electrons projected from the foil center reached the effective area of the MCP detector in the case of 170 Gauss. The simulation results support that the detection efficiency could be improved by reducing the transport time of the electrons from the foil to the MCP detector with a strong magnetic field.

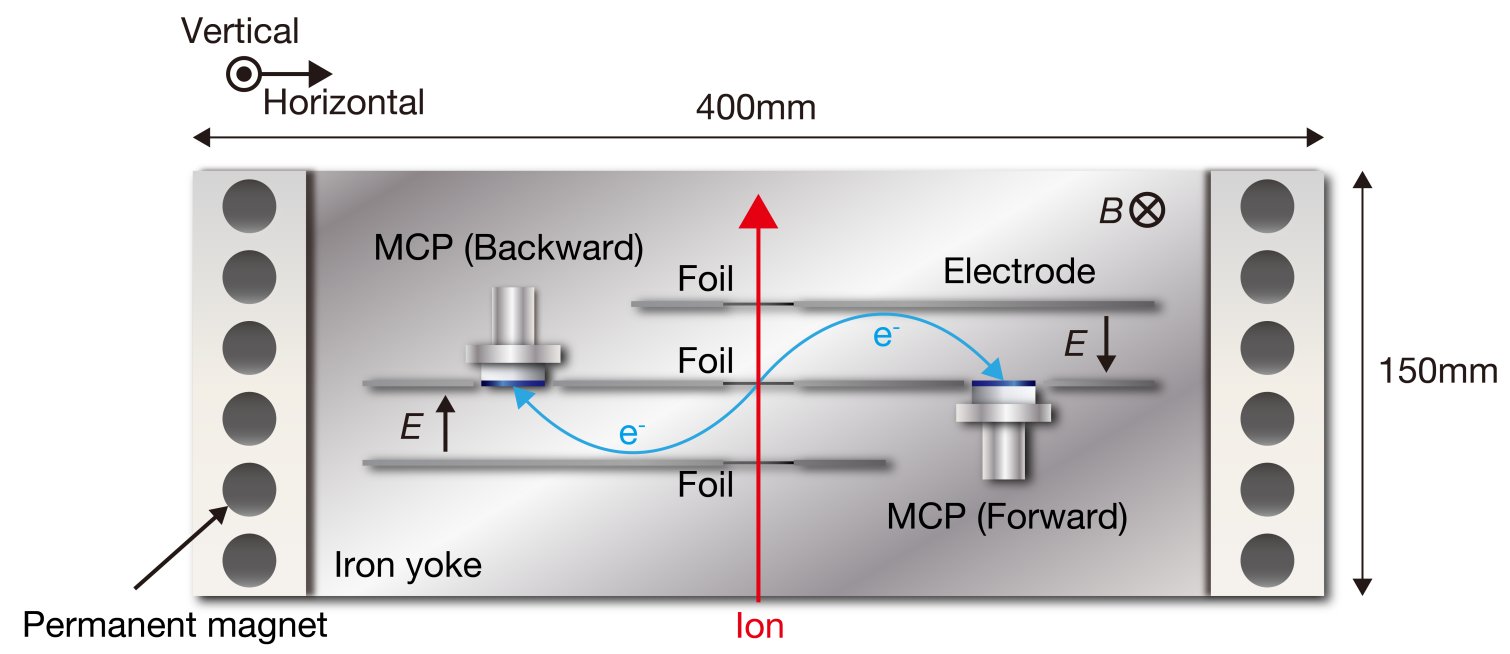

Figure 1: Schematic top view of the present time detector. In this figure, the upper iron yoke is not drawn as a matter of convenience.
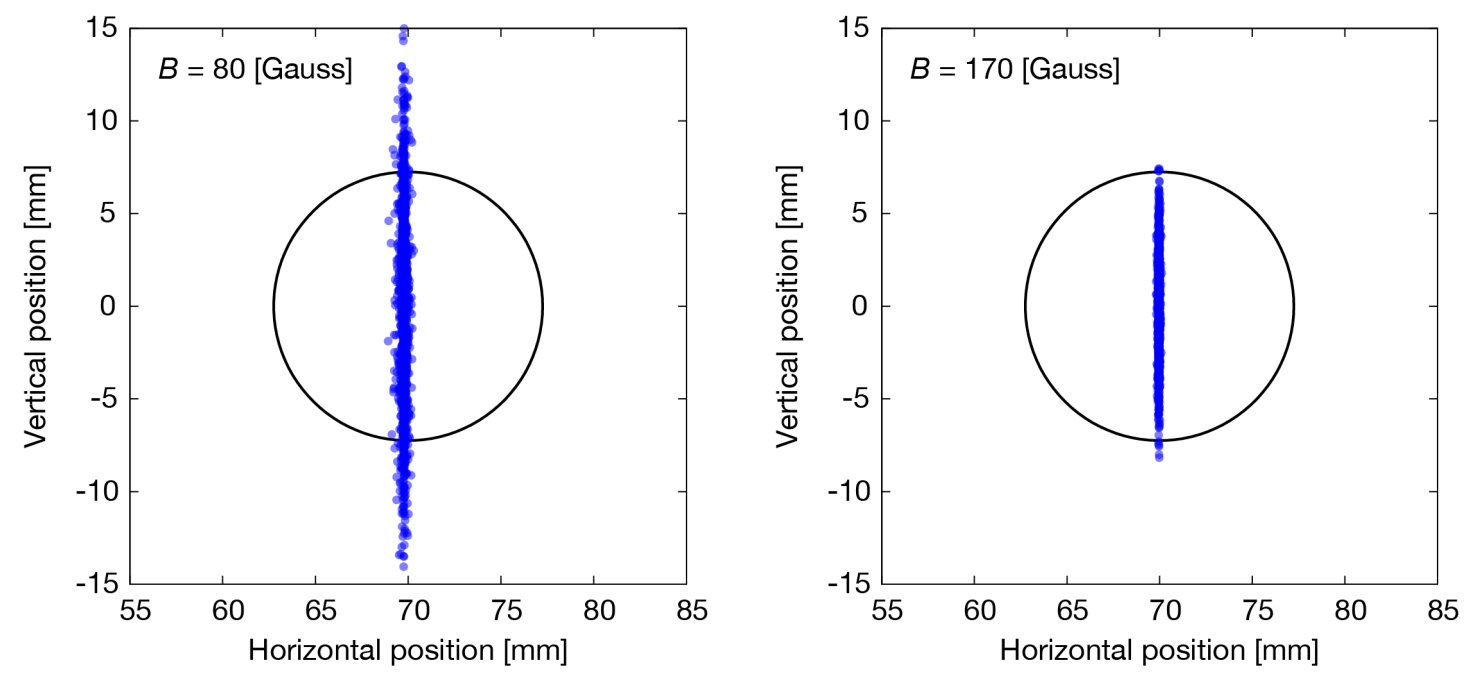

Figure 2: Two dimensional plots of the simulated electron positions at the plane which is in contact with the surface of the MCP detector showing the result at 80 Gauss (left) and 170 Gauss (right). Electron positions are shown by solid small circles. Open large circles indicate the effective area size of the MCP detector. Foil center is the origin of both coordinates. 


\section{Experimental performance with ion beam}

An experiment for the performance tests of the time detector was carried out with the ${ }^{84} \mathrm{Kr}$ beam of $200 \mathrm{MeV} /$ nucleons provided by HIMAC (Heavy Ion Medical Accelerator in Chiba) in National Institute of Radiological Sciences (NIRS). Figure B shows a schematic view of the experimental setup. The plastic scintillation counter consists of a scintillator and two Photo Multiplier Tubes (PMTs) which are connected to both sides of the scintillator. The coincidence signal of both PMTs was used as a trigger signal for data acquisition. Two Parallel Plate Avalanche Counters (PPACs) [18], position sensitive gas detectors, were used to measure, by extrapolation, the ion's position when passing through the foil. A sodium iodide scintillation counter was used to count ions which passed through the foil. The relative TOF of ions from the plastic scintillation counter to the time detector was measured by using Time to Analog Converter (TAC).

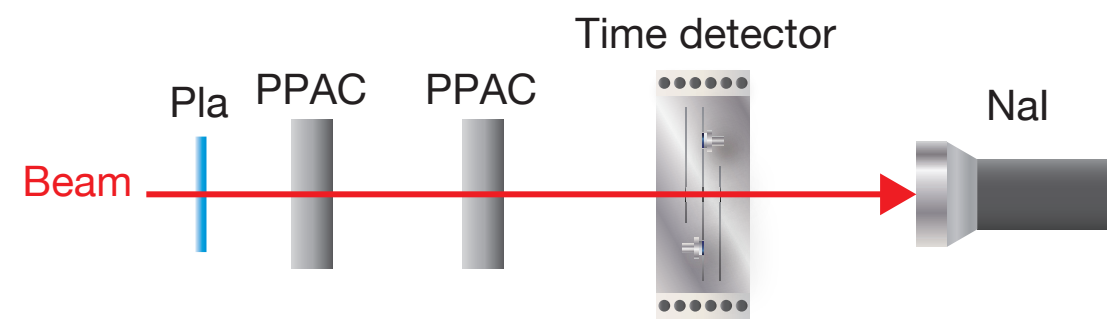

Figure 3: Schematic view of the experimental setup. The Pla, PPACs, and NaI show a plastic scintillation counter, parallel plate avalanche counters, and a sodium iodide scintillation counter, respectively.

The TOF histograms for the forward detection and the backward detection, are shown in Fig.⿴囗十. These histograms were measured by using the carbon foil as a secondary electron generator. In this histograms, the beam position is limited to the foil center $( \pm 1.3 \mathrm{~mm})$. Time resolution $\sigma$ were 30.4(5) ps and 31.9(5) ps for the forward and backward detection, respectively. These values include the intrinsic time resolution of the plastic scintillation counter. The time resolution $\sigma$ was less than $100 \mathrm{ps}$, for both the carbon and the gold coated Mylar. Figure $\square$ shows the position dependence of the relative TOF in the case of carbon foil. The difference of the maximum TOF and the minimum TOF was kept roughly within 100 ps for both the horizontal and the vertical position. It is influenced by the none uniformity of the electric field and the magnetic field.

The maximum efficiency of $99 \%$ was obtained at about $606 \mathrm{~V} / \mathrm{mm}$, for both the forward detection and the backward detection. In the case of gold coated Mylar, nearly the same results are obtained. Efficiency close to unity was achieved for both the carbon and the gold coated Mylar. Due to excessive energy loss in the gold coated Mylar without enhanced efficiency, a thin carbon foil is of better use for our purpose. Figure 6 shows the position dependence of the detection efficiency measured by using the carbon foil. In the wide region, high detection efficiency was obtained for both the forward and the backward detection. It is concluded that a sufficient amount of electrons emitted from any position of the foil was transported to the MCP detector. This result strongly supports that the reduction of the transport time of the electrons from the foil to the MCP detector using a strong magnetic field could improve the detection efficiency of the time detector. 

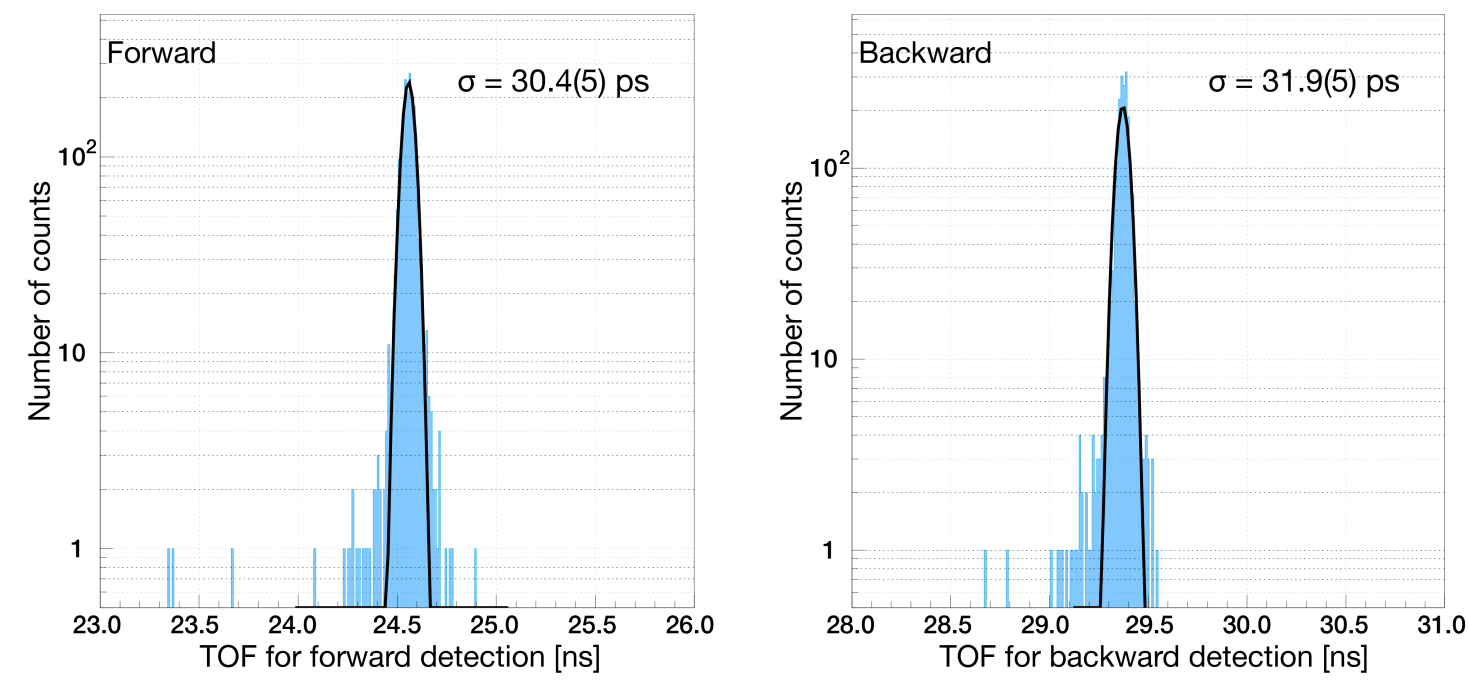

Figure 4: TOF histograms for the time detector. Left histgram shows the forward detection and the right histogram shows the backward detection. The solid lines indicate curve fit with Gaussian function.
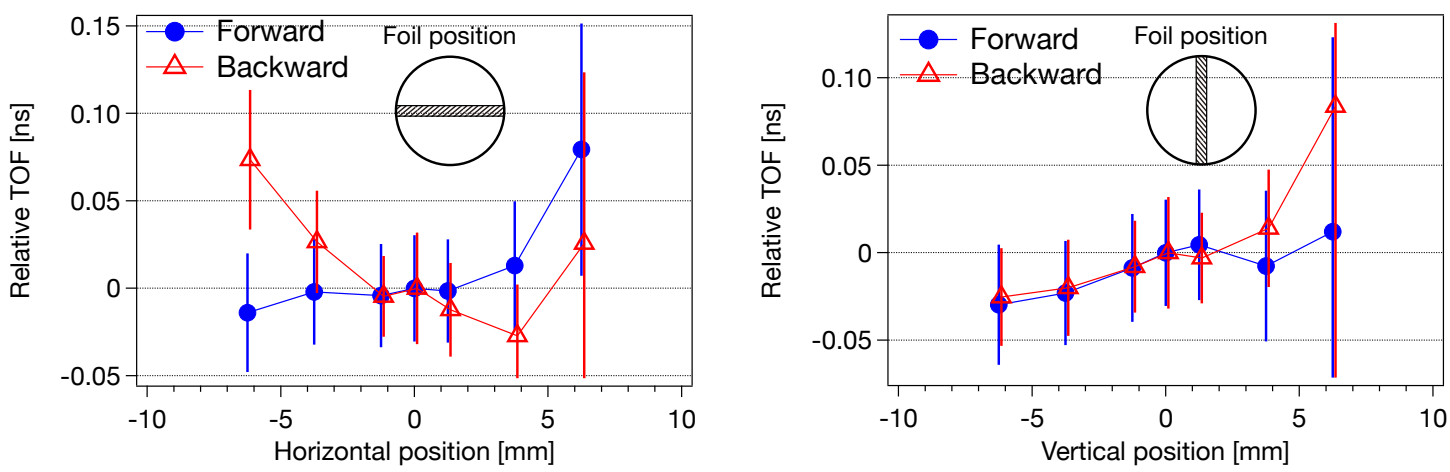

Figure 5: Position dependence of the relative TOF shown for horizontal (left) and vertical (right) position. TOF at foil center is assumed to be zero. Solid circles show the forward detection and the open triangles show the backward detection.
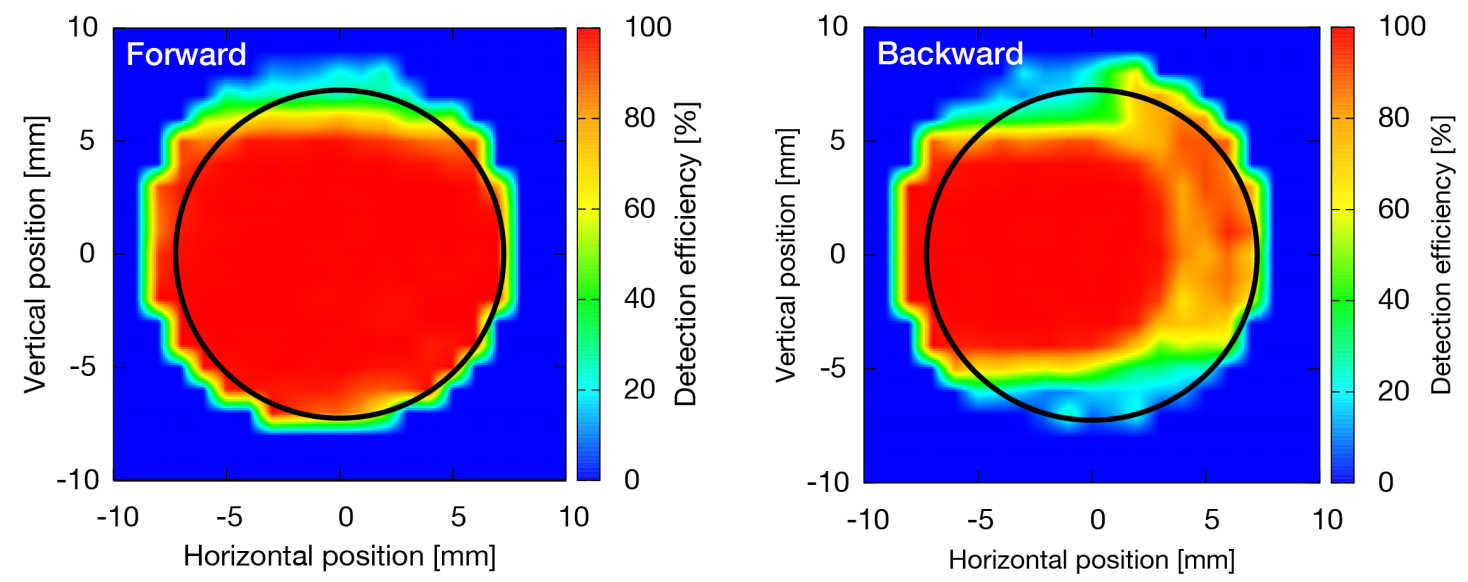

Figure 6: Two dimensional plots of the detection efficiency showing the result of forward detection (left) and the backward detection (right). Horizontal/vertical axis indicates horizontal/vertical position at the foil. The open circles show the effective area size of the MCP detectors. 


\section{Summary and further development}

The development of a time detector for the mass measurement with the Rare-RI Ring, is in progress. To improve the detection efficiency of the detector, the transport time of electrons is reduced by a strong magnetic field. The present prototype detector had satisfactory time resolution and the sufficient detection efficiency for heavy ion beams. There was no serious position dependence of the time performance and the detection efficiency. It is concluded that the reduction of the transport time of the electrons from the foil to the MCP detector is important to obtain the sufficient detection efficiency.

Except for detection acceptance, the present detector satisfied the requirements of mass measurements with the Rare-RI Ring. The expected beam size at the focal plane where the time detector will be installed, is about $\phi 30 \mathrm{~mm}$. Therefore, the time detector which satisfies the acceptance requirement is needed. A large acceptance detector which utilizes a combination of larger carbon foil and larger MCPs ( $\phi 42 \mathrm{~mm}$ ) is being developed for mass measurement at RIBF.

\section{Acknowledgments}

We would like to thank the accelerator staff of NIRS-HIMAC for providing the stable beam. This work was supported by Research Project with Heavy Ions at NIRS-HIMAC and JSPS KAKENHI Grant number 15H00830.

\section{References}

[1] M. Hausmann et al., Nucl. Inst. Meth. A 446 (2000) 569.

[2] J. Stadlmann et al., Phys. Lett. B 586 (2004) 24.

[3] B. Sun et al., Nucl. Inst. Meth. A 812 (2008) 1.

[4] J.W. Xia et al., Nucl. Inst. Meth. A 488 (2002) 11.

[5] X.L. Tu et al., Chin. Phys. C 33 (2009) 516.

[6] A. Ozawa et al., Prog. Theor. Exp. Phys. 03 C009 (2012).

[7] J. D. Bowman and R. H. Heffner, Nucl. Inst. Meth. 148 (1978) 503 .

[8] J. Trötscher et al., Nucl. Instr. and Meth. B 70 (1992) 455.

[9] M. Hausmann et al., Hyperfine Interact. 132 (2001) 291.

[10] N. K. Feuerstein et al., Nucl. Inst. Meth. A 821 (2016) 160.

[11] B. Mei et al., Nucl. Inst. Meth. A 624 (2010) 109.

[12] W. Zhang et al., Nucl. Inst. Meth. A 756 (2014) 1.

[13] D. Nagae et al., Nucl. Inst. Meth. B 317 (2013) 640.

[14] H. Rothard et al., Phys. Rev. A 41 (1990) 2521.

[15] A. Clouvas et al., Phys Rev. B 89 (1989) 6316.

[16] http://www.hamamatsu.com/jp/en/index.html

[17] C. G. Drexler and R. D. DuBois, Phys. Rev. A 53 (1996) 1630.

[18] H. Kumagai et al., Nucl. Inst. Meth. A 470 (2001) 562. 